\title{
Ultrafast nonlinear dynamics in thin GaN films studied by femtosecond digital holography
}

\author{
N. Šiaulys ${ }^{1}$, A. Urniežius ${ }^{2}$, T. Stanislauskas ${ }^{2}$, T. Malinauskas ${ }^{2}$, V. Kudriašov ${ }^{2, a}$, and A. Melninkaitis ${ }^{2, b}$ \\ 1 Laser Research Center, Vilnius University, Sauletekio 10, 10223 Vilnius, Lithuania \\ 2 Institute of Applied Research, Vilnius University, Sauletekio 10, 10223 Vilnius, Lithuania
}

\begin{abstract}
We studied the nonlinear propagation dynamics of intense $300 \mathrm{fs}$ infrared pulses in thin $\mathrm{GaN}$ films by use of pulsed digital holography technique with $25 \mathrm{fs}$ temporal resolution.
\end{abstract}

\section{Introduction}

Nonlinear propagation of ultrashort pulses in transparent materials is an active research field with many remarkable phenomena and applications [1]. Vast majority of studies consider the pulse propagation in conventional media like solids, liquids or gases. On the other hand, there is increasing interest in nonlinear interactions in artificial materials and waveguide structures suitable for all-optical applications [2,3]. Among various media $\mathrm{GaN}$ is considered to be one of the most prospective materials for these future nonlinear photonic applications [4]. However, to efficiently exploit its properties, a better understanding of the interplay between linear and nonlinear effects is needed, requiring more sophisticated methods to detect the processes occurring on ultrafast temporal scales. Recently, a pulsed digital holography was introduced as a new and prospective method for the study of complex ultrafast phenomena in dielectrics [5]. It combines best features of the pump-probe and digital holography techniques enabling one to detect very small phase perturbations of transmitted light in time and space resolved manner. In this work we use pulsed digital holographic method to study nonlinear response of thin $\mathrm{GaN}$ film to intense $300 \mathrm{fs}$ radiation of $1030 \mathrm{~nm}$ wavelength. For this purpose a holographic microscope with 25 fs temporal and better than $2 \mu \mathrm{m}$ spatial resolution was implemented. Due to the high temporal and spatial resolution two regions of positive and negative nonlinear change in refractive index were resolved in excitation zone. Furthermore the phonon-like oscillations were observed in the exited region for the first time when using this method. From the obtained digital holograms linear refractive indices were estimated for $o$ and $e$ polarizations.

\section{Experimental}

GaN film of $13.5 \mu \mathrm{m}$ thickness were grown on 2 inch diameter and $330 \mu \mathrm{m}$ thickness sapphire substrate by using metal-organic chemical vapor deposition (MOCVD). In order to prepare original GaN sample for our experiments we cleaved it into several parts. Cleaved edges were polished and the regions of the best optical quality served as an entrance surface of pump pulses in the experiment. The experimental scheme is demonstrated in Fig. 1. As a pump source a femtosecond $\mathrm{Yb}: \mathrm{KGW}$ laser ("Pharos", Light Conversion) operating at $1.03 \mu \mathrm{m}$ wavelength and generating $300 \mathrm{fs}$ duration pulses was used. Ultrafast response in GaN film was studied by focusing fundamental pulses into the polished

\footnotetext{
a e-mail: viaceslav.kudriasov@ff.vu.lt
}

b e-mail: andrius.melninkaitis@ff.vu.lt

This is an Open Access article distributed under the terms of the Creative Commons Attribution License 2.0, which permits unrestricted use, distribution, and reproduction in any medium, provided the original work is properly cited. 


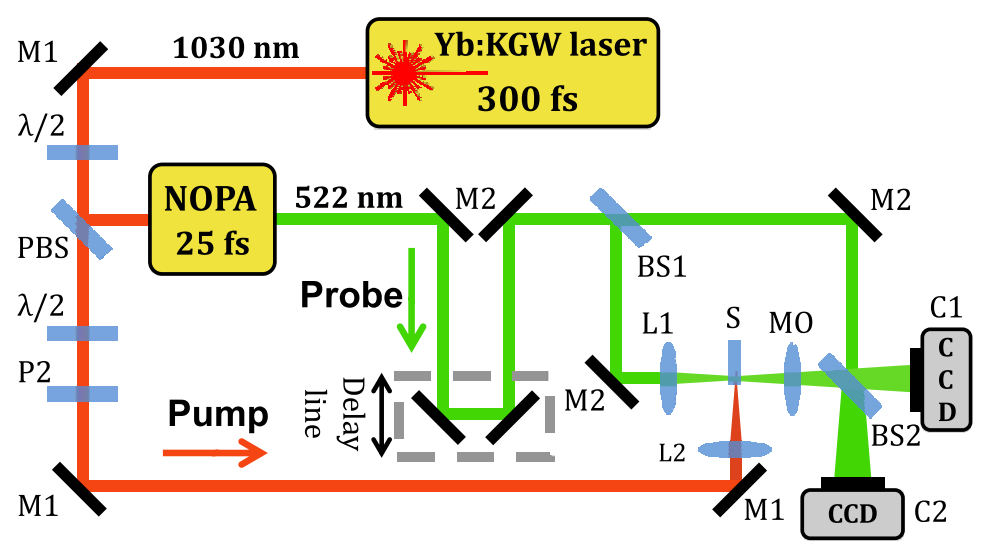

Fig. 1. Experimental setup demonstrating pump channel (red) and probe channel (green). Holography scheme is based on a Mach-Zehnder interferometer in off-axis configuration.

edge of the coating down to the $13.4 \mu \mathrm{m}$ (1/e level). A microscopic image of photo-induced "shadow" was captured holographically in transverse geometry by using Mach-Zehnder interferometer. Short probe pulses centered at $522 \mathrm{~nm}$ wavelength were generated by the non-collinear parametric amplifier (NOPA), which was pumped by the third harmonic of the fundamental radiation. The amplified output pulses were compressed by a prism pair to the 25 fs duration. By changing the energy and polarization of the pump pulse and its relative delay to the probe pulse the nonlinear dynamics inside the GaN film was studied. Polarization of the probe pulse was always parallel to the pump pulse propagation direction, while the polarization of the pump was changed.

\section{Results}

The response of $\mathrm{GaN}$ to the propagating pulses was studied for pump fluence ranging from $0.08 \mathrm{~J} / \mathrm{cm}^{2}$ to $1 \mathrm{~J} / \mathrm{cm}^{2}$, being close to the materials damage threshold. Amplitude and phase contrast images of the photo- induced "shadow" objects in GaN waveguide were reconstructed from the experimentally registered holograms and are shown in Fig. 2. These images clearly indicate well separated regions of refractive index changes induced during nonlinear propagation of the $300 \mathrm{fs}$ pump pulse $(1030 \mathrm{~nm})$. More specifically, the effects of self-focusing in the leading part of the pulse (negative phase shift) and the plasma defocusing (positive phase shift) at its trailing part are seen in the phase contrast images (Fig. 2b). The self-focusing is attributed to the non-resonant Kerr effect, as the photon energy of the pump pulse $(1.2 \mathrm{eV})$ is much lower than that of the $\mathrm{GaN}$ band-gap $(3.3 \mathrm{eV})$. The self-focusing region is accompanied by the positive phase change attributed to the generation of free electron plasma at higher pulse fluencies. It is seen that only phase contrast images (Fig. 2b) are able to show the areas of the reduced refractive index change due to the electron plasma, while the amplitude contrast images (Fig. 2a) show only the Kerr induced refractive index change. Also, the phase contrast images indicate some interesting features of the electron generation dynamics like its longitudinal and transverse modulation. The frequency of modulation observed in longitudinal direction corresponds to $9.3 \mathrm{THz}$ and is possibly attributed to phonon-like oscillations of the material density. However, more detail extensive study should be conducted in order to understand these effects. Furthermore, the effect of phase change saturation of the pulse is observed from the same series images in the leading part of the self-focused pulse, which can be attributed to the intensity clamping effect already observed in dielectrics [6]. Phase images also allow a direct evaluation of the nonlinear refractive index change induced by $1030 \mathrm{~nm}$ pulses from the phase change at $522 \mathrm{~nm}$ probe pulse wavelength. The obtained nonlinear refractive index change are of different sign and magnitude along the propagation direction. By changing delay time between the pump and probe pulses a direct observation of the pump pulse propagation was made. This data allowed a direct calculation of the refractive index for ordinary and 
(a)

(b)
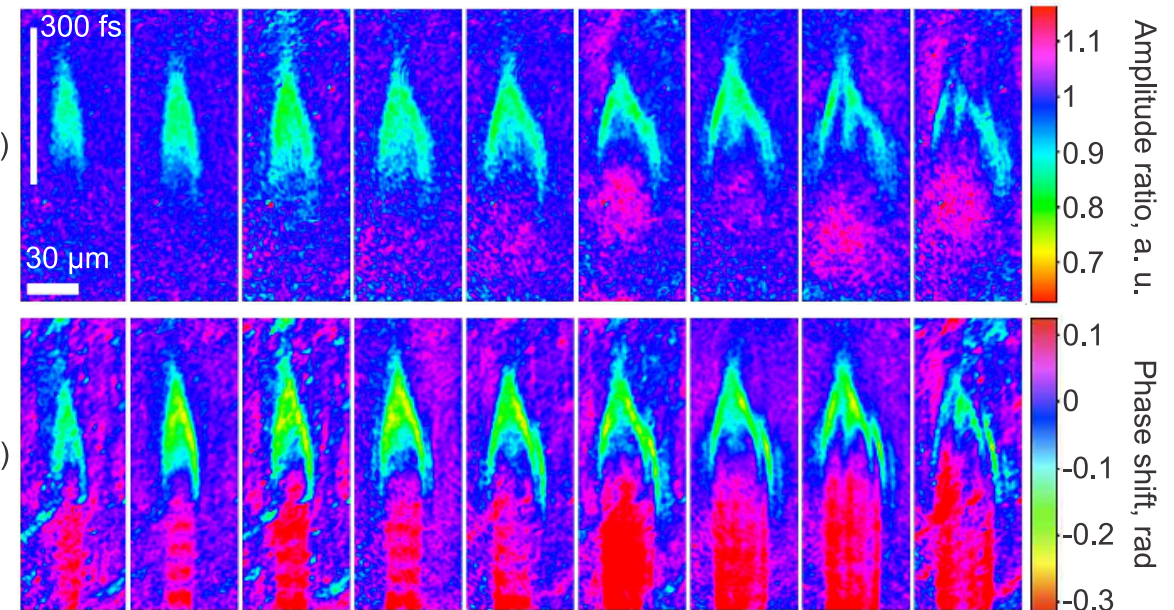

0.08

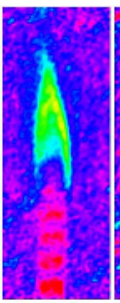

0.15

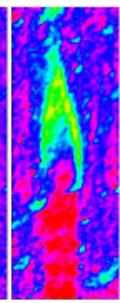

0.22

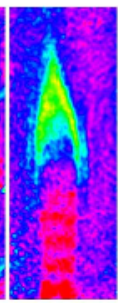

0.27

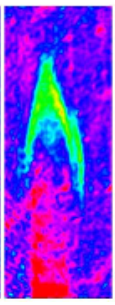

0.41

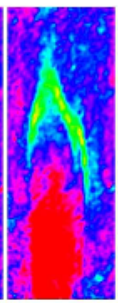

0.52

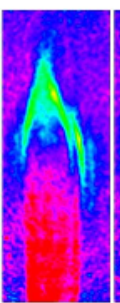

0.62

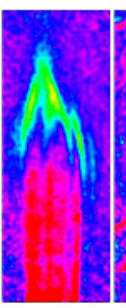

0.76

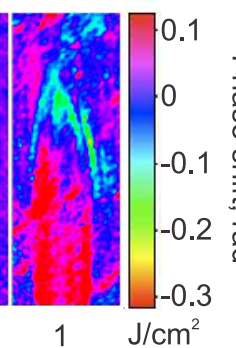

Fig. 2. Amplitude (a) and phase (b) contrast images reconstructed from the probe pulse digital holograms in GaN film for different pump pulse energy densities.

extraordinary polarization directions $\left(n_{o}=2.45\right.$ and $\left.n_{e}=2.54\right)$ at $1030 \mathrm{~nm}$ wavelength which are pretty close to those reported in literature.

\section{Conclusions}

For the first time nonlinear propagation of femtosecond pulses in thin GaN crystalline films were studied by using pulsed digital holography. Holographic microscope with 25 fs temporal and better than $2 \mu \mathrm{m}$ spatial resolution (both in amplitude and phase) was implemented and used for this study. From the obtained digital holograms the induced refractive index changes depending on the energy and polarization of the pump pulse were calculated. Direct observations demonstrate a wide range of interesting nonlinear effects occurring in GaN film at high intensity: Kerr effect, free electron generation, self-focusing and defocusing of propagating pulse as well as induced modulations in $\mathrm{THz}$ frequency domain. These effects can be clearly distinguished only in the phase images. In overall, it is demonstrated that femtosecond digital holography is a versatile tool well suited for the investigation of a wide range of effects taking place in transparent semiconductors or dielectrics.

\section{Acknowledgements}

This work has been supported by the Research Council of Lithuania, grant Nr. MIP-105/2011.

\section{References}

1. A. Couairon and A. Mysyrowicz, Phys. Rep. 441, (2007) 47.

2. R. Dekker, N. Usechak, M. Forst, and A. Driessen, J. Phys. D: Appl. Phys. 40, (2007) R249.

3. H. Ishikawa, ed., Ultrafast All-Optical Signal Processing Devices (Wiley, 2008) 258.

4. B. Gil, ed., Group III Nitride Semiconductor Compounds (Oxford University Press, 1998) 470.

5. T. Balciunas, A. Melninkaitis, G. Tamosauskas, and V. Sirutkaitis, Opt. Lett. 33, (2008) 58.

6. V.P. Kandidov, V.Yu. Fedorov, O.V. Tverskoy, O.G. Kosareva, and S.L. Chin, Quantum Electron. 41, (2011) 382 . 